\title{
Accuracy, reliability, feasibility and nurse acceptance of a subcutaneous continuous glucose management system in critically ill patients: a prospective clinical trial
}

Tobias Wollersheim ${ }^{1,2 \dagger}$, Lilian Jo Engelhardt ${ }^{1 \dagger}$, Jeanne Pachulla ${ }^{1}$, Rudolf Moergeli ${ }^{1}$, Susanne Koch ${ }^{1}$, Claudia Spies ${ }^{1}$, Michael Hiesmayr ${ }^{3}$ and Steffen Weber-Carstens ${ }^{1,2^{*}}$ (D)

\begin{abstract}
Background: Continuous glucose monitoring (CGM) has not yet been implemented in the intensive care unit (ICU) setting. The purpose of this study was to evaluate reliability, feasibility, nurse acceptance and accuracy of the Medtronic Sentrino ${ }^{\circledR}$ CGM system in critically ill patients.

Methods: Sensors were inserted into the subcutaneous tissue of the patient's thigh, quantifying interstitial glucose concentration for up to $72 \mathrm{~h}$ per sensor. Reliability and feasibility analysis included frequency of data display, data gaps and reasons for sensor removal. We surveyed nurse acceptance in a questionnaire. For the accuracy analysis, we compared sensor values to glucose values obtained via blood gas analysis. Potential benefits of CGM were investigated in intra-individual analyses of factors, such as glycemic variability or time in target range achieved with CGM compared to that achieved with intermittent glucose monitoring.

Results: The device generated 68,655 real-time values from 31 sensors in 20 critically ill patients. 532 comparative blood glucose values were collected. Data were displayed during $32.5 \mathrm{~h}$ [16.0/62.4] per sensor, which is $45.1 \%$ of the expected time of $72 \mathrm{~h}$ and $84.8 \%$ of $37.9 \mathrm{~h}$ actual monitoring time. 21 out of 31 sensors were removed prematurely. $79.1 \%$ of the nursing staff rated the device as not beneficial; the response rate was one-third. Mean absolute relative difference was $15.3 \%$ (Cl 13.5-17.0 \%). Clarke error grid: $76.9 \%$ zone A, $21.6 \%$ zone B, $0.2 \%$ zone C, 0.9 \% zone D, $0.4 \%$ zone E. Bland-Altman plot: mean bias $+0.53 \mathrm{mg} / \mathrm{dl}$, limits of agreement +64.6 and $-63.5 \mathrm{mg} / \mathrm{dl}$. Accuracy deteriorated during elevated glycemic variability and in the hyperglycemic range. There was no reduction in dysglycemic events during CGM compared to $72 \mathrm{~h}$ before and after CGM. If CGM was measuring accurately, it identified more hyperglycemic events when compared to intermittent measurements. This study was not designed to evaluate potential benefits of CGM on glucose control.
\end{abstract}

Conclusions: The subcutaneous CGM system did not perform with satisfactory accuracy, feasibility, or nursing acceptance when evaluated in 20 medical-surgical ICU patients. Low point accuracy and prolonged data gaps

\footnotetext{
*Correspondence: steffen.weber-carstens@charite.de

${ }^{\dagger}$ Tobias Wollersheim and Lilian Jo Engelhardt contributed equally to this work

${ }^{1}$ Department of Anesthesiology and Operative Intensive Care Medicine, Campus Charité Mitte and Campus Virchow Klinikum, Charité

- Universitätsmedizin Berlin, Augustenburger Platz 1, 13353 Berlin, Germany

Full list of author information is available at the end of the article
} provided you give appropriate credit to the original author(s) and the source, provide a link to the Creative Commons license, and indicate if changes were made. 
significantly limited the potential clinical usefulness of the CGM trend data. Accurate continuous data display, with a MARD $<14 \%$, showed potential benefits in a subgroup of our patients.

Trial registration NCT02296372; Ethic vote Charité EA2/095/14

Keywords: Continuous glucose monitoring, Subcutaneous, Interstitial, Critically ill patients, ICU, Medtronic Sentrino ${ }^{\circledR}$, Accuracy, Reliability, Feasibility, Nurse acceptance, Evaluation

\section{Background}

Critically ill patients frequently experience stress-induced alterations in glucose homoeostasis resulting in hyperglycemia [1]. Peripheral insulin resistance and an enhanced hepatic glucose production, caused by a release of counterregulatory hormones and cytokines, are contributing mechanisms $[1,2]$. Insufficient GLUT4 translocation in skeletal muscle of critically ill patients is related to glucose dysregulation [3]. Hyperglycemia, elevated glycemic variability and hypoglycemia, were associated with an increased mortality risk in critically ill patients [4-6]. Randomized controlled trials showed that insulin therapy and management of glycemic control in the ICU remains challenging [6-10].

Continuous glucose monitoring (CGM) in the ICU, combined with an appropriate insulin protocol, may improve management of glycemic control and consequently impact patient outcome [11-13]. Wernerman et al. provided an overview of CGM technologies, including glucose oxidase, mid-infrared spectroscopy and fluorescence, ranging from invasive intravascular devices to minimally invasive interstitial and noninvasive transcutaneous systems [11]. Interstitial devices designed for use in diabetic patients have already been applied in critically ill patients [13-16]. Despite promising attempts, these systems have not yet been implemented to daily routine in the ICU and improvements are desirable. The subcutaneous Medtronic Sentrino ${ }^{\circledR}$ CGM system was designed for use in ICU patients. The displayed real-time glucose trend line allows the ICU staff to observe glucose excursions at an earlier stage when compared to the established intermittent measurements. Patients may benefit from increased time in target range and improved glycemic variability. In addition, nurse workload may be reduced.

The purpose of this study was to evaluate reliability, feasibility, nurse acceptance and accuracy of this subcutaneous CGM system, as well as to identify potential weaknesses of the device in severely ill patients. In addition to previous studies [17-19], we retrospectively assessed potential benefits of CGM in comparison with intermittent glucose monitoring in our medical-surgical ICU.

\section{Methods}

\section{Inclusion criteria and study participants}

Inclusion criteria included an expected length of stay in the ICU of at least $72 \mathrm{~h}$, age $\geq 18$ years and written informed consent given by patient or legal proxy. We recruited critically ill patients during a time period of seven months in 2014. Patient inclusion started immediately after the local ethics committee, Ethikkommission Charité Universitätsmedizin Berlin, approved the study protocol (Charité-EA2/095/14). The protocol was registered under https://clinicaltrials.gov, trial registration number NCT02296372.

\section{Glycemic control in the study setting}

The single-center study was set in two interdisciplinary mixed medical-surgical ICUs of a university hospital. The glucose target levels for insulin therapy were $80-149 \mathrm{mg} /$ dl. Dysglycemic events were defined as follows: ranges above $149 \mathrm{mg} / \mathrm{dl}$ represented moderate hyperglycemia, and glucose levels above $179 \mathrm{mg} / \mathrm{dl}$ represented severe hyperglycemia. Moderate hypoglycemia was defined in a range from 41 to $70 \mathrm{mg} / \mathrm{dl}$, and severe hypoglycemia as $\leq 40 \mathrm{mg} / \mathrm{dl}[11,20]$. Due to general ICU routine, nurses took blood samples from an arterial catheter in 2- to 4-h intervals, depending on the patient's condition. In the absence of an arterial line, blood was collected from a central or peripheral venous catheter. Blood glucose was determined by glucose oxidase reaction using a Radiometer ABL 800 FLEX (Copenhagen, Denmark) blood gas analyzer. Depending on the identified blood glucose value, the nursing staff regulated the intravenous insulin therapy according to the local insulin protocol (Additional file 1: Table S1). All patient data, including blood gas analyses, were documented within the patient data management system (PDMS).

\section{CGM sensor}

According to manufacturer's information, the sensors of the interstitial CGM device consist of four independently working electrodes, which are embedded in two cannulas. This multisensory system provides enhanced signal stability and accuracy in critically ill patients. The electrodes are coated by glucose oxidase. In the enzymatic reaction, electrons are released and create an electrical gradient, which is proportional to the interstitial glucose concentration. Based on the electrical signal, the CGM algorithm calculates out of the four data signals one blood glucose value, which is displayed on a bedsided monitor. The device provides one real-time glucose 
measurement per minute, with an insignificant lag time for signal processing, for up to $72 \mathrm{~h}$ (for more details, see Additional file 1: CGM Device).

\section{Study procedure}

We inserted the sensors into the subcutaneous tissue of patient's upper leg. After initialization, the sensors required one initial blood glucose entry, followed by two further calibrations after the first and second running hour. Subsequently, the study team performed calibrations every $8 \mathrm{~h}$, as proposed by the manufacturer. The ward staff were not required to perform further calibrations. We instructed nurses to observe the continuous glucose trend line and perform blood glucose measurements to adapt insulin therapy in case of excursions above or below the target range (defined in the local insulin protocol Additional file 1: Table S1). Glucose values determined by the blood gas analyzer were used as reference. Blood glucose measurements used for initial calibrations and calibrations after data gaps (>15 $\mathrm{min}$ ) were excluded from the point accuracy analysis. Further blood glucose measurements were included and compared to the latest CGM value immediately before calibration. As specified in the study protocol (Fig. 1), the accuracy analysis required a minimum monitoring time of $48 \mathrm{~h}$ or at least 12 comparative readings.

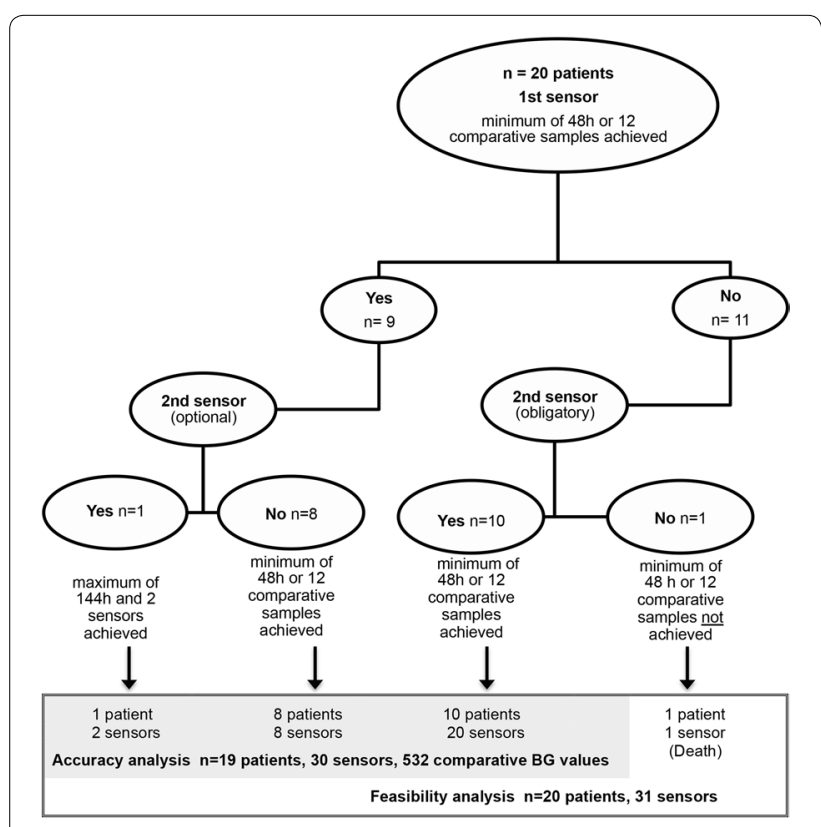

Fig. 1 Study procedure. We included $n=20$ patients during 57 days of recruiting. One patient was excluded from the accuracy analysis due to a lack of comparative blood glucose samples. Ten patients required a second sensor to achieve the minimum number of comparative samples or a minimum running time of $48 \mathrm{~h}$. We used an optional second sensor in one patient

\section{Analysis criteria}

Figure 2 illustrates detailed endpoints for the analysis of reliability, feasibility, nurse acceptance, accuracy and potential benefits of CGM. The analysis is based on the 2013 consensus recommendations, published by Finfer et al. defining criteria for continuous glucose control in critically ill patients [20]. Desirable reliability criteria include a continuous data display during $>95 \%$ of time and device-related data gaps $<30 \mathrm{~min}$ [20]. We calculated frequency of data gaps and analyzed the gaps subdivided as very brief $(<15 \mathrm{~min})$, brief $(15-30 \mathrm{~min})$, prolonged $(>30 \mathrm{~min})$ and very prolonged $(>2 \mathrm{~h})$, so as to better describe the clinical significance of the missing trend data. The feasibility analysis considered the capacity of the device to perform within the busy ICU setting. This was supplemented by a survey of nurse acceptance assessed by brief questionnaires given to the nurses in charge of each shift (Additional file 1: Fig. S3). To determine accuracy, sensor values were compared to the simultaneously recorded blood glucose values from PDMS. We calculated point accuracy according to criteria specified within the consensus recommendations [20], which can be summarized as follows

- $98 \%$ of device readings should be within $12.5 \%$ of a reference standard (or within $\pm 10 \mathrm{mg} / \mathrm{dl}$ for readings $<100 \mathrm{mg} / \mathrm{dl}$ )

- The remaining $2 \%$ of readings should be within $20 \%$ of a reference standard

- Mean absolute relative difference (MARD) should be $<14 \%(\mathrm{M}) \mathrm{ARD}=\mid$ (blood glucose - sensor glucose) $\mid$ /blood glucose $\times 100$

- MARD > $18 \%$ represents poor accuracy.

In addition, we analyzed possible confounding factors on MARD, such as arterial $\mathrm{pO}_{2}$, temperature, hemoglobin, potassium, lactate, $\mathrm{pH}$ value, sequential organ failure assessment (SOFA) Score, systemic inflammatory response syndrome (SIRS), history of diabetes, blood glucose variability and glucose ranges $(<80 \mathrm{mg} /$ dl, $80-179 \mathrm{mg} / \mathrm{dl},>179 \mathrm{mg} / \mathrm{dl}$ ). We retrospectively calculated MARD after time-shifting the reference a fixed amount (1 up to $30 \mathrm{~min}$ ), so as to investigate a time delay as a possible confounding factor. To investigate potential benefits of CGM in our ICU, we report glycemic control achieved with CGM compared to that achieved with intermittent glucose monitoring. This was accomplished by performing intra-individual analyses, longitudinal and parallel, of factors such as mean blood glucose level, blood glucose variability, number of dysglycemia events and time in blood glucose target range (Fig. 2). Glycemic variability was determined using standard deviation of blood glucose and glycemic lability index (Table $4 \mathrm{~b}$ ), as a 


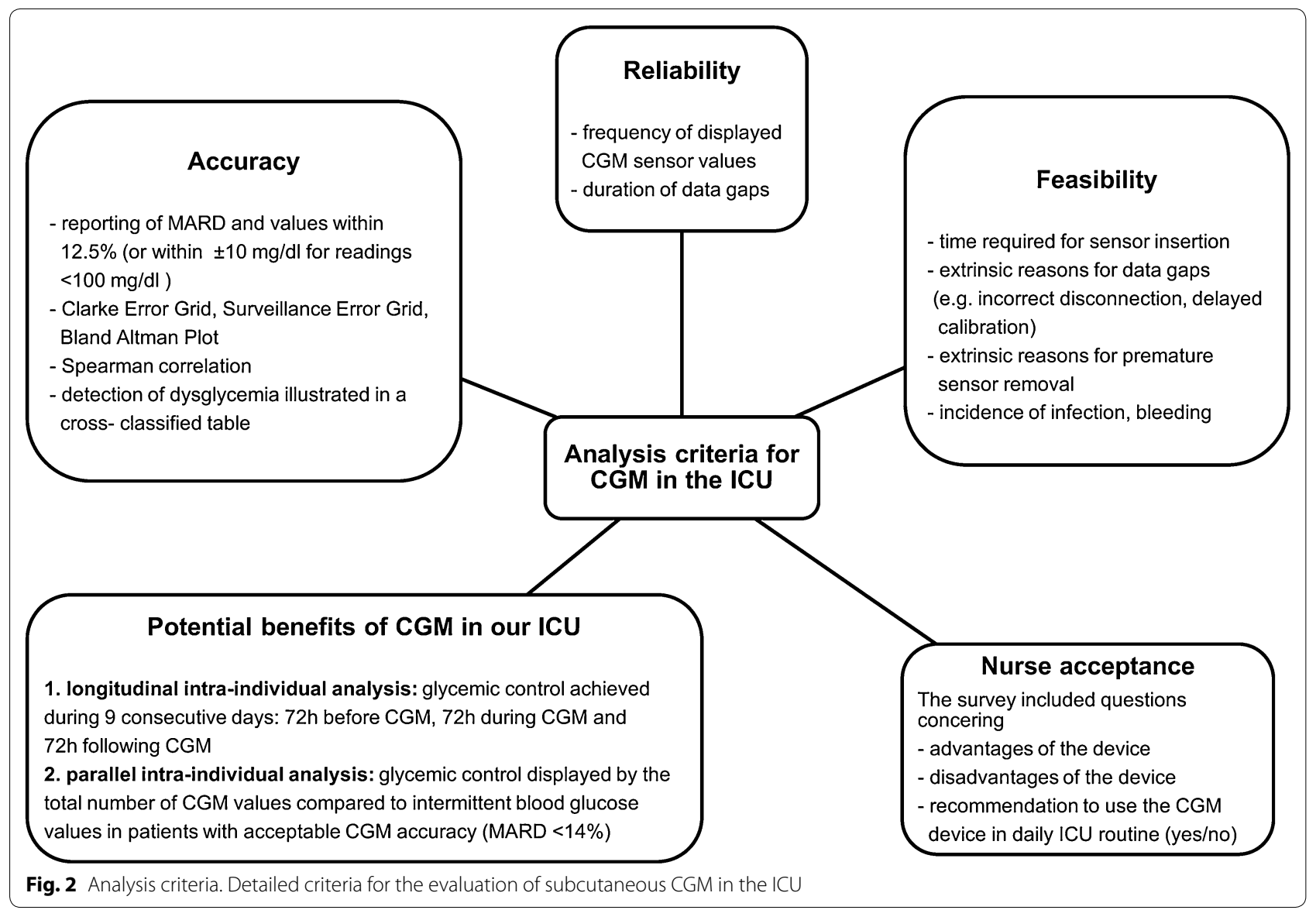

time-weighted index [21]. To evaluate safety, we reported local complications and discussed patient risks due to inaccurate CGM measurements in a safety statement.

\section{Statistical analysis}

Results were shown as median with interquartile range or as absolute numbers with percentages. Clinical accuracy was illustrated using Bland-Altman plot [22], Clarke error grid [23] and Surveillance Error-Grid [24]. We calculated glycemic lability index using EasyGV৫ software [21]. Nonparametric tests were performed (Mann-Whitney $U$ Test, Wilcoxon Test, Kruskal-Wallis Test, Friedman Test and Spearman's correlation). We used IBM॰ SPSS $\odot$ Statistics version 21, Microsoft Excel๔ ${ }^{2010}$ and R for the statistical analysis.

\section{Results}

We included 20 critically ill patients in this prospective trial using a total of 31 sensors (Fig. 1). Table 1 shows patient characteristics. In total, the device generated 68655 (1144.3 $\mathrm{h}$ ) real-time glucose values during $1337.1 \mathrm{~h}$ of monitoring. The median monitoring time per patient was $70.5 \mathrm{~h}$ [57.2/72.7]. For the accuracy comparison, we collected 532 blood glucose values in 19 patients, of which 475 (89.3\%) were obtained from arterial and 57 (10.7 \%) from venous catheters. There was no significant difference in accuracy between the 475 arterial blood glucose values compared to all 532 glucose values $(p=.799)$. Table 2 shows a summary of glycemic control metrics.

\section{Reliability, feasibility and safety}

The reliability analysis showed a real-time data display during $32.5 \mathrm{~h}(16 / 62.4)$ per sensor, which is $45.1 \%$ of the expected time of $72 \mathrm{~h}$ and $84.8 \%$ of the $37.9 \mathrm{~h}$ actual monitoring time. During $80223 \mathrm{~min}(1337.1 \mathrm{~h})$ of monitoring, we observed in total $11568 \mathrm{~min}(192.8 \mathrm{~h})$ of missing values. The number of data gaps was 155 , of which 68 (43.9\%) were very brief (<15 $\mathrm{min}), 35(22.6 \%)$ were brief (15-30 $\mathrm{min}), 27$ (17.4\%) were prolonged (30-120 min) and $25(16.1 \%)$ were very prolonged. The sensor insertion itself was easily performed and required less than $10 \mathrm{~min}$. The complication rate at the site was low. Minor bleeding after insertion occurred in four patients. We observed no local infection. The main feasibility issue was premature sensor removal. Detailed device reliability and feasibility is shown in Table 3. 
Table 1 Patient characterization

\begin{tabular}{ll}
\hline Age & $61[54 / 69]$ \\
Gender (female/male) & $14(70 \%) / 6(30 \%)$ \\
BMI (kg/m ${ }^{2)}$ & $23[22 / 26]$ \\
Diagnosis leading to ICU stay & $6(30 \%)$ \\
ARDS (ECMO, ECLA) & $8(40 \%)$ \\
ARDS (without ECMO, ECLA) & $1(5 \%)$ \\
Mediastinitis & $3(15 \%)$ \\
Peritonitis & $1(5 \%)$ \\
Intracranial hemorrhage & $1(5 \%)$ \\
Polytrauma & $20(100 \%)$ \\
At least one event of SIRS or sepsis during CGM & $8[4 / 10]$ \\
Sequential organ failure assessment (SOFA) Score at inclusion & $24[19 / 28]$ \\
Acute physiology and chronic health evaluation (APACHE) 2 Score at admission & $5(25 \%)$ \\
History of diabetes mellitus & $14(70 \%)$ \\
Administration of intravenous insulin therapy during CGM & $7(35 \%)$ \\
Administration of vasopressors during CGM & $0.08[0.03 / 0.14]$ \\
Mean dose of epinephrine during sensor running time $(\mu \mathrm{gg} / \mathrm{kg} / \mathrm{min})($ in seven patients receiving vasopressors) & $4(20 \%)$ \\
Mortality during ICU stay &
\end{tabular}

\section{$n=20$ patients}

Results are expressed as median with interquartile range or as absolute numbers with percentages of $n=20$ patients

$B M I$ body mass index, ARDS acute respiratory distress syndrome, ECMO extracorporeal membrane oxygenation, ECLA extracorporeal lung assist, SIRS systemic inflammatory response syndrome, SOFA sequential organ failure assessment, APACHE acute physiology and chronic health evaluation, ICU intensive care unit, CGM continuous glucose monitoring

\section{Table 2 Mean glucose level, glycemic variability and glycemic events}

\begin{tabular}{|c|c|c|}
\hline & Reference blood glucose & Comparative CGM reading \\
\hline Number of comparative glucose readings & 532 & 532 \\
\hline Readings per patient & $28[18 / 34]$ & $28[18 / 34]$ \\
\hline Mean glucose level per patient (mg/dl) & $133.8[128.4 / 147.5]$ & $133.7[124.3 / 150.1]^{*}$ \\
\hline $\begin{array}{l}\text { Glucose variability per patient measured in } \\
\text { SD (mg/dl) }\end{array}$ & $24.8[19.9 / 35.2]$ & $32.5[25.2 / 42.2]^{\S}$ \\
\hline \multicolumn{3}{|c|}{ Glycemic events: number and percentage of $n=19$ patients } \\
\hline Severe hypoglycemia ( $\leq 40$ mg/dl) & $1(5.3 \%)$ & $1(5.3 \%)$ \\
\hline Moderate hypoglycemia (41-70 mg/dl) & $1(5.3 \%)$ & $10(52.6 \%)$ \\
\hline Euglycemia (71-149 mg/dl) & $19(100 \%)$ & $19(100 \%)$ \\
\hline Moderate hyperglycemia (150-179 mg/dl) & $18(94.7 \%)$ & $19(100 \%)$ \\
\hline Severe hyperglycemia (>179 mg/dl) & $15(78.9 \%)$ & $11(57.9 \%)$ \\
\hline $\begin{array}{l}\text { Glycemic events: number and percentage of } \\
n=532 \text { readings }\end{array}$ & $158(29.7 \%)$ & $188(35.3 \%)$ \\
\hline Severe hypoglycemia ( $\leq 40$ mg/dl) & $1(0.2 \%)$ & \multirow{5}{*}{$\begin{array}{l}\text { Chi-square test: } p<0.001 \text { for crosstabulation see } \\
\text { supplement }\end{array}$} \\
\hline Moderate hypoglycemia (41-70 mg/dl) & $2(0.4 \%)$ & \\
\hline Euglycemia (71-149 mg/dl) & $374(70.3 \%)$ & \\
\hline Moderate hyperglycemia (150-179 mg/dl) & $96(18.0 \%)$ & \\
\hline Severe hyperglycemia (>179 mg/dl) & $59(11.1 \%)$ & \\
\hline
\end{tabular}

$n=19$ patients

Results are expressed as median with interquartile range or as absolute numbers with percentages

$S D$ standard deviation

${ }^{*} p=1.0 ;{ }^{\S} p=0.002$ 
Table 3 Reliability and feasibility

\begin{tabular}{|c|c|c|c|c|}
\hline & & & & Per sensor \\
\hline \multicolumn{3}{|l|}{ Initialization time } & & $27 \mathrm{~min}$ \\
\hline \multicolumn{3}{|l|}{ Total time until first displayed value } & & $37.5 \min [36 / 42]$ \\
\hline \multicolumn{3}{|l|}{ Expected monitoring time after initialization } & & $72 \mathrm{~h}$ \\
\hline \multicolumn{3}{|l|}{ Actual monitoring time after initialization } & & $37.9 \mathrm{~h}[23 / 71.3]$ \\
\hline \multicolumn{3}{|l|}{ Real-time data display } & & $32.5 \mathrm{~h}[16 / 62.4]$ \\
\hline \multicolumn{3}{|c|}{ Percentage of real-time data display/expected monitoring time after initialization } & & $45.1 \%$ \\
\hline \multicolumn{3}{|c|}{ Percentage of real-time data display/actual monitoring time after initialization } & & $84.8 \%[67.9 / 93.8]$ \\
\hline \multicolumn{3}{|l|}{ Data gaps after initialization } & & 5 h $[1.9 / 8.3]$ \\
\hline \multicolumn{3}{|c|}{ Percentage of data gaps/actual monitoring time after initialization } & & $15.2 \%[6.2 / 32.1]$ \\
\hline \multicolumn{3}{|l|}{ Number of performed calibrations } & & $9.5[6 / 13]$ \\
\hline $\begin{array}{l}\text { Reasons for the } 11,568 \mathrm{~min} \\
(192.8 \mathrm{~h}) \text { of data gaps }\end{array}$ & \multicolumn{2}{|c|}{$\begin{array}{l}\text { Percentage of data gaps } \\
11,568 \mathrm{~min}(192.8 \mathrm{~h})\end{array}$} & \multicolumn{2}{|c|}{$\begin{array}{l}\text { Percentage of monitoring } \\
\text { time } 80,223 \mathrm{~min}(1337.1 \mathrm{~h})\end{array}$} \\
\hline 1 Poor sensor signal (\%) & \multicolumn{2}{|l|}{23.3} & \multicolumn{2}{|l|}{3.4} \\
\hline 2 Sensor failure (\%) & \multicolumn{2}{|l|}{15.0} & \multicolumn{2}{|l|}{2.2} \\
\hline 3 Processor line error (\%) & \multicolumn{2}{|l|}{10.9} & \multicolumn{2}{|l|}{1.6} \\
\hline 4 Disconnection (\%) & \multicolumn{2}{|l|}{15.6} & \multicolumn{2}{|l|}{2.3} \\
\hline 5 Pending after reconnection (\%) & \multicolumn{2}{|l|}{3.4} & \multicolumn{2}{|l|}{0.5} \\
\hline 6 Calibration required (\%) & \multicolumn{2}{|l|}{27.8} & \multicolumn{2}{|l|}{4.0} \\
\hline 7 Others (\%) & \multicolumn{2}{|l|}{4.0} & \multicolumn{2}{|l|}{0.6} \\
\hline Device-related reasons (1-3) (\%) & \multicolumn{2}{|l|}{49.2} & \multicolumn{2}{|l|}{7.2} \\
\hline Not device related (4-7) (\%) & \multicolumn{2}{|l|}{50.8} & \multicolumn{2}{|l|}{7.4} \\
\hline Reasons for 21 prematurely sensor removals & $\begin{array}{l}\text { Number of } \\
\text { sensors }\end{array}$ & $\begin{array}{l}\text { Percentage of } \\
\text { all sensors (\%) }\end{array}$ & & $\begin{array}{l}\text { Percentage of } \\
\text { removed sensors (\%) }\end{array}$ \\
\hline Sensors 72 h completed & 10 & 32.3 & & - \\
\hline Sensors removed prematurely & 21 & 67.7 & & 100 \\
\hline 1 Accidentally & 7 & 22.6 & & 33.3 \\
\hline 2 Poor sensor signal during measurement & 7 & 22.6 & & 33.3 \\
\hline 3 Poor sensor signal immediately after initialization & 1 & 3.2 & & 4.8 \\
\hline $4 \mathrm{MRI}$ & 1 & 3.2 & & 4.8 \\
\hline 5 Discharge & 1 & 3.2 & & 4.8 \\
\hline 6 Death & 2 & 6.5 & & 9.5 \\
\hline 7 Others & 2 & 6.5 & & 9.5 \\
\hline Device-related reasons (1-3) & 15 & 48.4 & & 71.4 \\
\hline Not device related (4-7) & 6 & 19.4 & & 28.6 \\
\hline
\end{tabular}

$n=20$ patients, 31 sensors

Results are expressed as median with interquartile range or as absolute numbers with percentages

$M R I$ magnetic resonance imaging

\section{Nurse acceptance}

The nurses received 128 questionnaires during the CGM monitoring period. The response rate was one-third ( $n=43,34 \%)$. The majority (79.1\%) of the nursing staff rated the device as not beneficial in the daily ICU routine. Advantages, such as the opportunity to observe glucose trends, were reported in $20.9 \%$ of the questionnaires. Disadvantages were described by $53.5 \%$. Reasons included the inadequate alarm performance (23.3\%), the additional device $(23.3 \%)$ and device line $(6.9 \%)$ as disturbing factors during bedding and mobilization in the ICU routine.

\section{Point accuracy}

$60.3 \%$ of sensor data were within $12.5 \%$ from the reference blood glucose (or were within $\pm 10 \mathrm{mg} / \mathrm{dl}$ for readings $<100 \mathrm{mg} / \mathrm{dl}$ ). In total, $76.9 \%$ of sensor readings were within 20 , and $23.1 \%$ deviated more than $20 \%$ from the reference. MARD was $15.3 \%$ (95\% CI 13.5-17.0\%). Spearman's correlation coefficient was $0.688, p<.001$, 
$r^{2}=0.461$. The Bland-Altman plot (Fig. 3a) showed a mean bias of $0.53 \mathrm{mg} / \mathrm{dl}$ and limits of agreement of $+64.6 \mathrm{mg} / \mathrm{dl}$ and $-63.5 \mathrm{mg} / \mathrm{dl}$. Clarke error grid and Color-Coded Surveillance Error-Grid (Fig. 3b, c) showed potentially dangerous errors. Additional file 1: Table S2 shows the detection of dysglycemic events.

\section{Confounding factors on accuracy}

We identified that the blood glucose variability, analyzed in standard deviation, was significantly associated with CGM accuracy (Fig. 4a). Confirming this finding, standard deviation per patient was positively correlated with MARD per patient $k=0.593, p=.001, n=19, r^{2}=0.298$ (Additional file 1: Fig. S1). MARD deteriorated in the hyperglycemic blood glucose range (Fig. 4a). There was no significant improvement or deterioration of MARD after time-shifting the reference glucose a fixed amount of 1 up to $30 \mathrm{~min}$ (Additional file 1: Fig. S2). MARD was worse during application of vasoconstrictors (Additional file 1: Table S3a). Previously known diabetes mellitus and episodes of SIRS did not confound MARD (Additional file 1: Table S3a). The severity of disease, measured via SOFA Score, showed a minor positive correlation with the MARD $\left(k=0.088, p=.043, r^{2}=0.006, n=532\right)$. There was no significant correlation of arterial pO2, temperature, $\mathrm{pH}$ value, lactate, hemoglobin, or potassium and MARD (Additional file 1: Table S3b).

\section{Potential benefits of CGM in our ICU}

In 10 patients with an ICU stay of at least nine consecutive days, the longitudinal analysis showed no significant reduction in dysglycemic events during 3 days of CGM compared to $72 \mathrm{~h}$ before and $72 \mathrm{~h}$ after CGM (Table 4a). In the parallel analysis, CGM determined significantly lower minimal glucose values and detected more hyperglycemic events compared to intermittent blood glucose values in eight patients, in whom the device displayed accurate results with a MARD $<14 \%$ (Table 4b).

\section{Discussion}

\section{Reliability, feasibility and nurse acceptance}

This prospective study was initially conducted with the intention of implementing a minimally invasive, simple to use CGM device in our ICU, in order to improve glycemic control. Unfortunately, application and performance were not as reliable as expected. Numerous sensors were removed prematurely, and the percentage of data gaps in relation to the expected sensor running time exceeded the time specified within the consensus recommendations of ICU experts [20]. The fact that we did not demand additional calibrations from the nursing staff and that they were not involved in troubleshooting may have contributed to the extent of data gaps and the poor performance. Since $7.4 \%$ of data gaps were not device related, the data display during $85.6 \%$ of the sensor running time after initialization could be corrected to $93 \%$. The high rate of accidentally removed sensors underlines the vulnerable use of the subcutaneous device in intensive care. This is supported by the opinion of our ICU nursing staff. More experience with a device may enhance feasibility. However, device-related issues, which are not improvable by experience, occurred frequently. Recently published investigations evaluating the same device reported minimal differences in reliability, but the clinically relevant results were concordant [17-19].

\section{Point accuracy and confounding factors}

The subcutaneous device did not fulfill the suggested accuracy criteria for CGM in critically ill patients, specified within the consensus recommendations of ICU experts [20]. The distribution in the Clarke error grid [23] was unsatisfactory, as all 532 comparative readings of this analysis should have been located in zone A or B, preferably in zone A. The Surveillance Error-Grid [24], which promises to be closer to clinical routine, showed similar degrees of risk. In the Bland-Altman plot, the mean bias indicated that there was no systematic error [22]. However, $95 \%$ of the values were within $128 \mathrm{mg} / \mathrm{dl}$ of the reference glucose. These wide limits of agreement illustrated a high random error [22]. The detection of dysglycemia was critical. The results considering MARD values within the $12.5 \%$ range, and Clarke error grid and Bland-Altman plot are precisely consistent with those reported by Van Hooijdonk et al. [17]. Two further studies showed slightly better accuracy of the same system $[18,19]$. Although specifically designed for ICU use, the investigated subcutaneous device failed to achieve comparatively accurate results in all recently published trials [17-19], as opposed to the CGM technologies quantifying glucose concentration in the vascular compartment of critically ill patients [25-28]. This leads to the conclusion that, with the intention to administrate insulin therapy, the subcutaneous glucose determination is not the proper method to estimate blood glucose levels during the acute phase of severe illness.

Inaccuracies may be attributed to a physiological time delay relating to the glucose diffusion from the plasma to the interstitial compartment $[29,30]$. In healthy humans and diabetes patients, this time delay has been observed to range from 0 to $40 \mathrm{~min}$ in various studies summarized by Scuffi et al. [30]. Rebrin et al. found no evidence that physiological delays exceeded 5-10 min and argued that device-related processes are responsible for longer periods [31]. Moreover, Boyne et al. addressed the issue of random inter-sensor time discrepancies, which were quantitatively similar to physiological time delays, when 

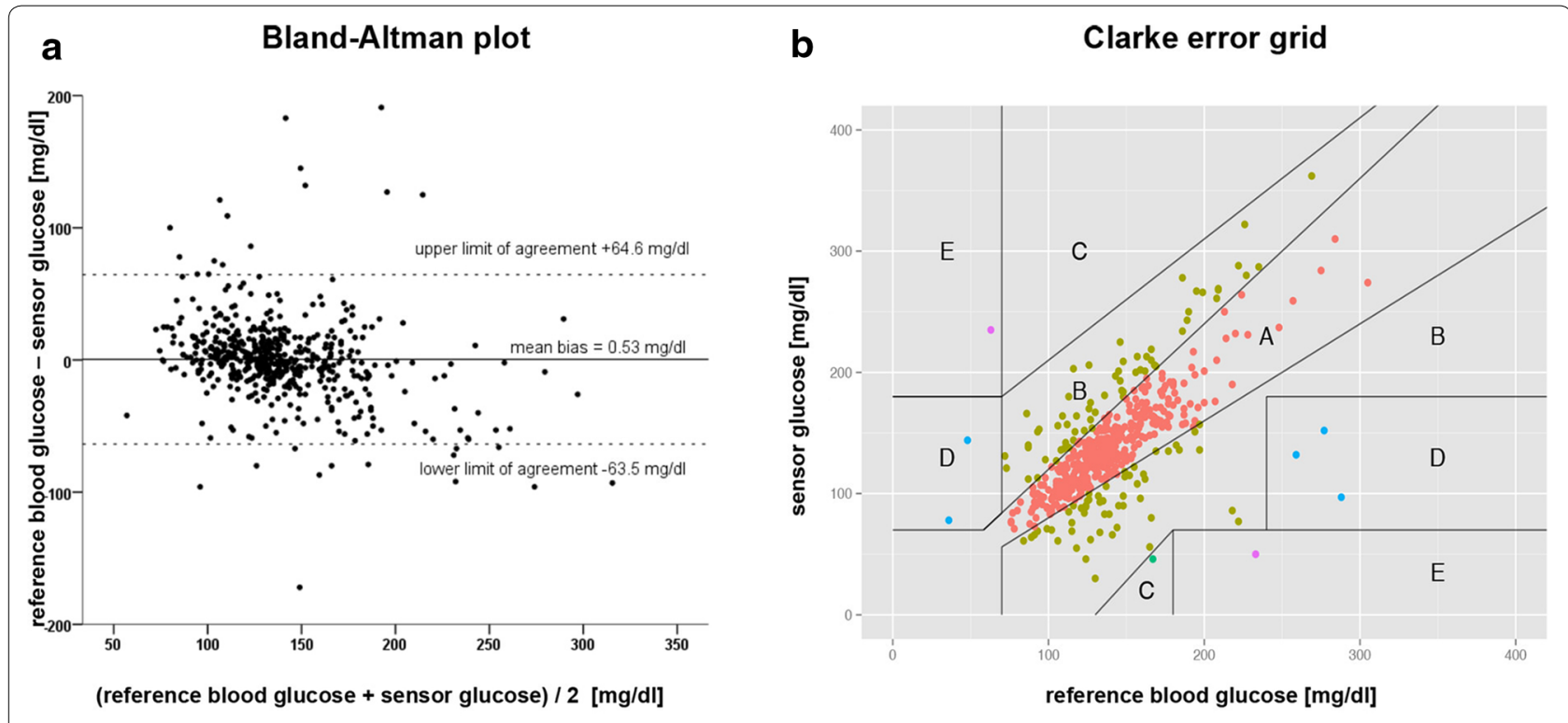

C

Color-Coded Surveillance Error-Grid

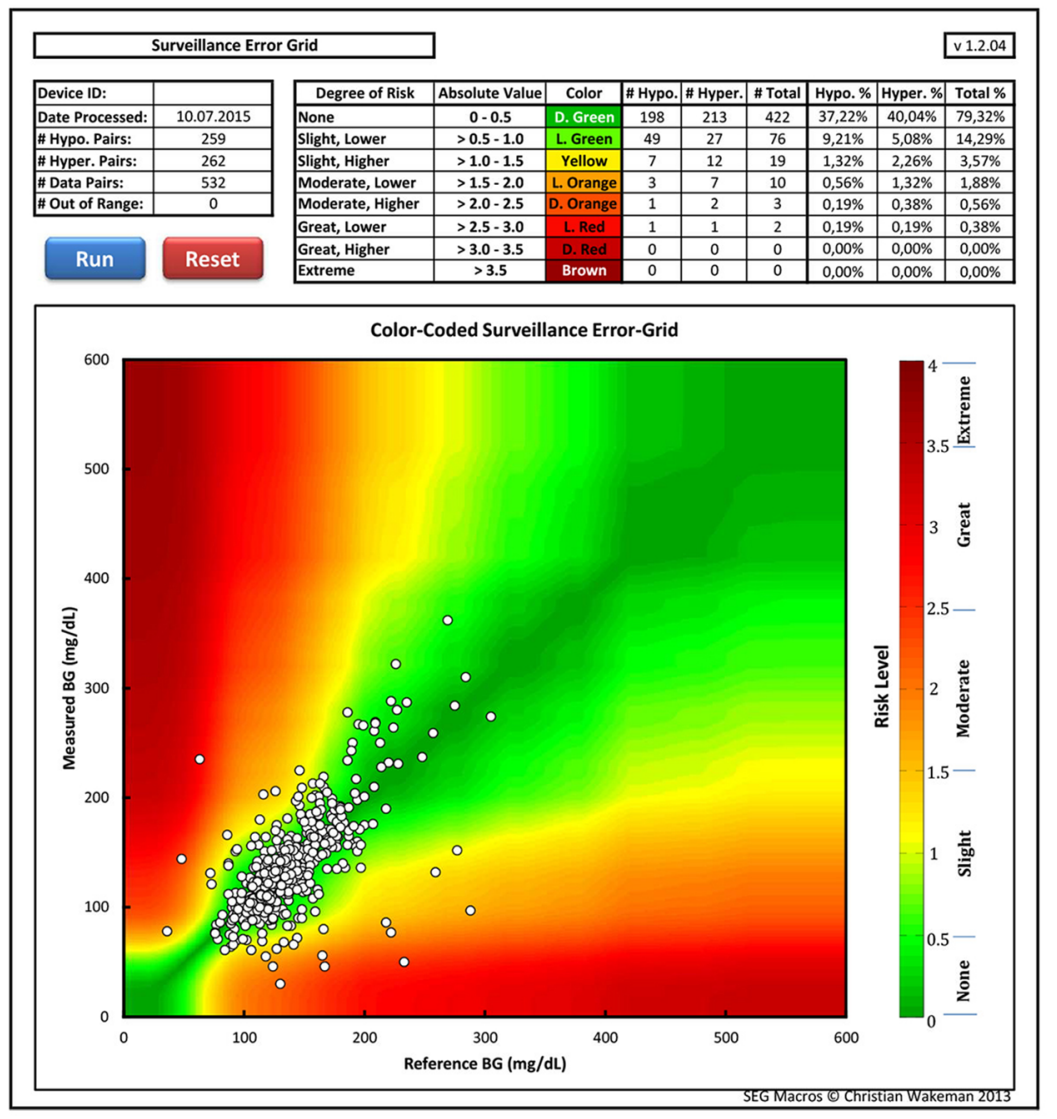


(See figure on previous page.)

Fig. 3 Bland-Altman plot, Clarke error grid, Color-coded Surveillance Error-Grid. $n=532$ comparative samples. a Bland-Altman plot. The mean bias indicates whether there is a systematic error. Upper and lower limits were calculated by mean bias $\pm 1.96 \times$ standard deviation of the difference between $B G$ and sensor glucose and represent random variations around the mean bias. If there is a Gaussian distribution, $95 \%$ of points are located between these limits. $[22,41]$. b Clarke error grid. Distribution: $A=76.9 \%, B=21.6 \%, C=0.2 \%, D=0.9 \%, E=0.4 \%$. Zones $A$ (CGM data $\leq 20 \%$ deviation from $B G$ ) and $B$ are considered as clinically acceptable zones, whereas values in zones $C, D$ and $E$ are increasingly dangerous for the patient, and zone E may lead to adverse therapeutic decisions. [23]. c Color-coded Surveillance Error-Grid. The Surveillance Error-Grid software is available at http://www.diabetestechnology.org/SEGsoftware/Surveillance-Error-Grid-Analysis.xlsm. Last Accessed: Dec 112015 [24]

comparing measurements of two subcutaneous sensors in the same individual [32]. Factors influencing the glucose diffusion rate, such as blood flow, peripheral microcirculation, and metabolic rate of subcutaneous tissue and adjacent cells, are all frequently altered in critically ill patients $[11,33]$. We found no indication for a fixed time shift, but time delay and interstitial sensor accuracy may vary depending on the patient's condition. There is evidence to support this hypothesis, since the use of vasopressors and a higher SOFA Score downgraded sensor accuracy in the present trial. In contrast, the accuracy of a subcutaneous CGM device was significantly improved in patients with septic shock compared to patients without sepsis [34]. Further studies cited that circulatory shock requiring norepinephrine therapy and impaired microcirculation had no influence on subcutaneous sensor accuracy $[35,36]$. Variable subcutaneous oxygen concentration may interfere with the glucose oxidase. We did not investigate tissue paO2, but arterial paO2, as a correlating factor, had no clinically relevant impact on accuracy.

Glucose homeostasis is affected by the peripheral glucose uptake [33]. Inflammation may lead to an insufficient GLUT 4 translocation to sarcolemmal membrane [3]. This mechanism resulted in an impaired glucose supply in skeletal muscle cells in ICU patients [3]. A decreased glucose uptake was observed in adipocytes of septic rats [37]. We hypothesize that an insufficient GLUT 4 translocation may occur in subcutaneous tissue cells of critically ill patients. This may influence the accuracy of a

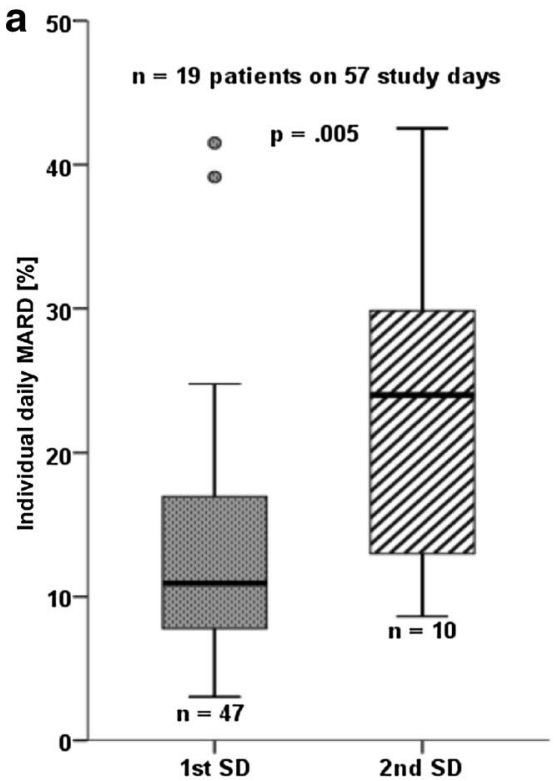

standard deviation (SD) of reference blood glucose 30.2 [mg/dl]

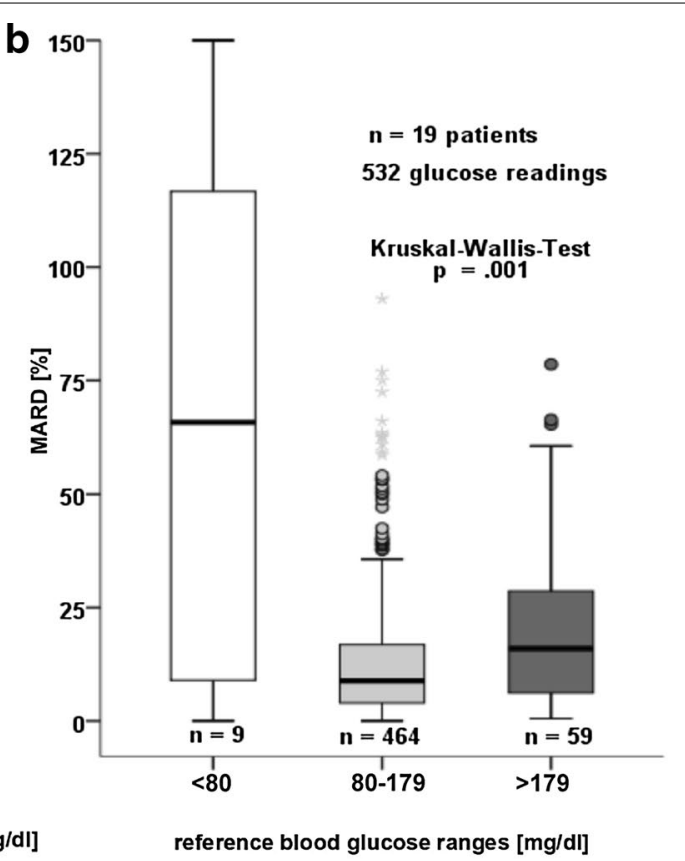

reference blood glucose ranges [mg/dl]

Fig. 4 Confounding factors on MARD. a (left): Association between MARD and individual daily blood glucose variability shown in first and second standard deviation of reference glucose. First boxplot The CGM device shows acceptable accuracy* (MARD median $10.9 \%$ ) if the blood glucose variability is low (first standard deviation). Second boxplot Accuracy deteriorates (MARD median $24 \%$ ) during increased blood glucose variability (second standard deviation). b (right): Association between MARD and blood glucose ranges. Second boxplot The CGM device shows acceptable accuracy* (MARD median $8.8 \%$ ) in blood glucose ranges between 80 and 179 mg/dl. First and Third boxplots Accuracy deteriorates in the hypoglycemic range (MARD median $65.8 \%$ ) and during severe hyperglycemia (MARD median $16 \%$ ). ${ }^{*}$ According to criteria specified within the consensus recommendations [20], MARD should be $<14 \%$ 
Table 4 Potential benefits of CGM in our ICU

\begin{tabular}{|c|c|c|c|c|}
\hline Per patient & $\begin{array}{l}\text { Blood glucose } 3 \text { days } \\
\text { before CGM }\end{array}$ & $\begin{array}{l}\text { Blood glucose } 3 \text { days } \\
\text { during } \text { CGM* }^{*}\end{array}$ & $\begin{array}{l}\text { Blood glucose } 3 \text { days } \\
\text { after CGM }\end{array}$ & $p$ value \\
\hline \multicolumn{5}{|c|}{$\begin{array}{l}\text { (a) Longitudinal analysis. Blood glucose } \\
\text { metrics before/during/after CGM. } n=10 \\
\text { patients, } 998 \text { blood glucose values }\end{array}$} \\
\hline Number of BGA & $30[25 / 40]$ & $35.5[28 / 41]$ & $26.5[22 / 34]$ & $p=.001^{\S}$ \\
\hline Number of hypoglycemia & $0[0 / 0]$ & $0[0 / 0]$ & $0[0 / 0]$ & $p=1.00$ \\
\hline Number of hyperglycemia & $3[1 / 4]$ & $2.5[1 / 5]$ & $2[1 / 3]$ & $p=.779$ \\
\hline Time in target (in \%) & $82.5[74 / 98.2]$ & 81.6 [68.9/95.6] & $88.7[81.3 / 94.3]$ & $p=.452$ \\
\hline Time < $>1$ mg/dl (in \%) & $0[0 / 0]$ & $0[0 / 0]$ & $0[0 / 0]$ & $p=1.00$ \\
\hline Time >149 mg/dl (in \%) & $17.5[1.8 / 26]$ & $18.4[4.4 / 31.1]$ & $11.3[5.7 / 15.4]$ & $p=.717$ \\
\hline Blood glucose min (mg/dl) & $89[80 / 100]$ & $85[73 / 106]$ & $97.5[85 / 110]$ & $p=.273$ \\
\hline Blood glucose max (mg/dl) & $173.5[162 / 187]$ & $202[159 / 218]$ & $166[153 / 185]$ & $p=.014^{\#}$ \\
\hline Mean glucose level (mg/dl) & $134[126.1 / 137.1]$ & $130.7[123.5 / 139]$ & $128.5[120.6 / 138.4]$ & $p=.497$ \\
\hline Mean glucose SD (mg/dl) & 18.9 [15.8/22.4] & $20.7[17.6 / 36.4]$ & $16.2[11.6 / 24.2]$ & $p=.741$ \\
\hline Per patient & & $\begin{array}{l}\text { Blood glucose values } \\
(n=239)\end{array}$ & $\begin{array}{l}\text { CGM sensor glucose } \\
(n=34056)\end{array}$ & $p$ value \\
\hline \multicolumn{5}{|c|}{$\begin{array}{l}\text { (b) Parallel analysis. Comparison of intermittent blood glucose to CGM } \\
\text { glucose metrics including the total number of CGM readings, } n=8 \\
\text { patients MARD }<14 \%, 239 \text { blood glucose values, } 32,044 \text { CGM values }\end{array}$} \\
\hline Number of readings & & $29.5[26.5 / 31.5]$ & 3975 [3780/4109] & $p=.012$ \\
\hline Number of hypoglycemia & & $0[0 / 0]$ & $0.5[0 / 2]$ & $p=.066$ \\
\hline Number of hyperglycemia & & $1[1 / 5]$ & $7[6 / 18]$ & $p=.018$ \\
\hline Time in target range (in \%) & & $88.7[60.7 / 96.5]$ & $85.2[57.9 / 91.6]$ & $p=.208$ \\
\hline Time < $>11$ mg/dl (in \%) & & $0[0 / 0]$ & $0.3[0 / 2.3]$ & $p=.068$ \\
\hline Time >149 mg/dl (in \%) & & $11.3[3.6 / 39.3]$ & $14.3[6.2 / 40.6]$ & $p=.327$ \\
\hline Glucose min (mg/dl) & & $103.5[87 / 1111.5]$ & $76[62 / 91]$ & $p=.017$ \\
\hline Glucose max (mg/dl) & & $195[154.5 / 211]$ & $186[178.5 / 220.5]$ & $p=.208$ \\
\hline Mean glucose level (mg/dl) & & $130.2[124.3 / 147.9]$ & 128.7 [120.5/147.4] & $p=.327$ \\
\hline Mean glucose SD (mg/dl) & & $19.9[14.4 / 22.7]$ & $20.6[16.5 / 28.4]$ & $p=.093$ \\
\hline Glycemic lability index & & $38.0[14 / 53]$ & 36.9 [18.5/90.7] & $p=.674$ \\
\hline
\end{tabular}

Intra-individual longitudinal and parallel analysis, target range $71-149 \mathrm{mg} / \mathrm{dl}$

Results are expressed as median with interquartile range or as absolute numbers with percentages

Glycemic lability index: time interval $1440 \mathrm{~min}=24 \mathrm{~h}$, glucose in $\mathrm{mg} / \mathrm{dl}$, sampling interval: blood glucose $120 \mathrm{~min}=2 \mathrm{~h}$, sensor glucose $1 \mathrm{~min}$

Number of hypoglycemia or hyperglycemia: only events of newly developed hypoglycemia or hyperglycemia were considered in the analysis

$B G A$ blood glucose analysis, SD standard deviation, GLI glycemic lability index

$\S$ Wilcoxon test before and during $p=.123$; before and after $p=.05$, during and after $p=.005$

\# Wilcoxon test before and during $p=.241$, before and after $p=.415$, during and after $p=.013$

* BGAs during CGM data gaps and times of temporary system failure are included

subcutaneous CGM device, when compared to blood glucose. Consequently, it can still be assumed that subcutaneous CGM reflects actual insulin-dependent tissue glucose dynamics, which may be clinically relevant [32].

We identified that the sensor accuracy deteriorated in patients with elevated glycemic variability, as well as in the hyperglycemic range. Unfortunately, inaccuracies of CGM occurred particularly often when the need for CGM would have been most beneficial. Delayed diffusion processes become increasingly significant during rapid glucose oscillations $[30,33]$ and may contribute to the adverse influence of glucose variability and hyperglycemia on sensor performance. In healthy volunteers, the interstitial glucose was similar to venous glucose during steady-state conditions, but an increased time delay was observed when glucose levels were rapidly elevated by glucose infusion [38]. We could not confirm the findings reported by van Hooijdonk et al. that accuracy was influenced by a history of diabetes [17]. As already assumed in this study, inaccuracies in critically ill diabetic patients were possibly attributable to glucose fluctuations [17]. Although intravascular and interstitial space should be 
considered as different glucose compartments, the sensor technology requires blood glucose calibrations [33, 39]. This is a major concern, since sensor calibration during glucose alterations may subsequently cause and amplify inaccuracies [33].

\section{Safety statement}

The local complication rate was acceptable, but critical safety issues arose as a consequence of inaccurate measurements. Clarke error grid and Surveillance Error-Grid showed potentially dangerous situations for the patients. Clinicians need to be aware of the fact that this device is not safe to guide insulin therapy. Even if used only to support common glucose control, this device can lead to confusing situations in the ICU routine of glucose management. As our experience showed, clinicians should always critically question the displayed CGM data.

\section{Potential benefits of CGM in our ICU}

Glucose monitoring with the CGM system did not improve glycemic control in the longitudinal, intraindividual analysis. Low accuracy, as well as low nurse acceptance, may be potential reasons. Besides, the time in target of our severely ill patients with and without CGM was high. As a consequence, it may be difficult to demonstrate improved control even with a device that had reasonable accuracy. Conversely, if CGM was accurate, it showed potential benefits. In contrast to the findings of Brunner et al., glycemic variability was not significantly different when calculated from accurate continuous values as compared to less frequent blood glucose values [40]. If accurate CGM systems and adapted insulin protocols are implemented in the ICU, further research is required to evaluate long-term effects on clinical outcomes in RCTs. Insulin therapy guided by CGM did not impact on time in target range and glycemic variability in previous RCTs $[13,14,40]$.

\section{Potential areas for improvements}

- Calibration should only be performed during "steadystate" glucose levels, and not during rapid glucose fluctuations $[33,39]$ or adapted within a special calibration algorithm

- Improved fixation method or different localization to avoid accidental sensor removal

- Wireless device to avoid data gaps caused by occasional disconnection during bedding or mobilization, as well as accidental removals

- Integration of the continuous glucose display into the established patient monitor to reduce additional equipment

- Inclusion of a suggestion according to the local insulin treatment protocol into monitor

\section{Limitations}

Firstly, this was a point accuracy analysis, in which only the concurrent blood glucose sample was considered. The reporting of glucose trending is not possible in this trial. Secondly, in the clinical setting we cannot exclude that there is a delay between the taking of a blood sample and the actual analysis via blood gas analyzer, where the time-point is documented [12, 20]. Thirdly, not all nurses were familiar with the device after the initial instructions provided by the manufacturer. Fourthly, the low response rate to the questionnaires may bias the results of the nurse acceptance survey. Fifthly, due to the low number of actual hypoglycemic events, there is a lack of evidence to draw a conclusion concerning the accuracy during hypoglycemia. It has to be stressed that this study was not designed to evaluate potential benefits of CGM on glucose control and there was no variation to the insulin protocol.

\section{Conclusion}

The Medtronic System did not perform with satisfactory accuracy, feasibility or nursing acceptance when evaluated in 20 medical-surgical ICU patients. Low point accuracy and prolonged data gaps significantly limited the potential clinical usefulness of the CGM trend data. Future studies are required to determine the clinical value of the real-time Sentrino ${ }^{\circledR}$ glucose trend data and alarms, using a validated nurse-driven insulin dosing algorithm in order to improve the safety and efficacy of blood glucose control in hospitalized patients.

\section{Additional file}

Additional file 1: Supplementary Method. CGM Device. Supplementary Tables. Table S1. The local insulin protocol. Table S2. Detection of dysglycemic events. Table S3a. Confounding factors on MARD. Table S3b. Spearman's correlation of paO2, temperature, lactate, pH-value, hemoglobin, potassium and SOFA-Score and MARD. Supplementary Figures. Fig. S1. Correlation of blood glucose variability per patient and MARD per patient. Fig. S2. MARD after time-shifting the reference a fixed amount (1 up to 30 minutes). Fig. S3. Nurse questionnaire.

\section{Abbreviations}

ICU: intensive care unit; BG: blood glucose; CGM: continuous glucose monitoring; Cl: confidence interval; ICU: intensive care unit; MARD: mean absolute relative difference; PDMS: patient data management system; RCT: randomized controlled trial; SOFA: sequential organ failure assessment.

\section{Authors' contributions}

TW and LE contributed equally to this work, designed the study, performed data acquisition, analyzed and interpreted data and wrote the manuscript. JP performed data acquisition, analyzed nurse acceptance and critically revised the manuscript for important intellectual content. RM critical revised the manuscript and gave input on calculation of glycemic variability. SK, CS, $\mathrm{MH}$ critically revised the manuscript for important intellectual content. SWC revised the manuscript, performed statistical analysis and made substantial contributions to data interpretation. All authors read and approved the final manuscript. 


\begin{abstract}
Author details
1 Department of Anesthesiology and Operative Intensive Care Medicine, Campus Charité Mitte and Campus Virchow Klinikum, Charité - Universitätsmedizin Berlin, Augustenburger Platz 1, 13353 Berlin, Germany. ${ }^{2}$ Berlin Institute of Health (BIH), Berlin, Germany. ${ }^{3}$ Division Cardiac-, Thoracic-, Vascular Anesthesia and Intensive Care, Medical University Vienna, Vienna, Austria.
\end{abstract}

\section{Acknowledgements}

Preliminary data of this manuscript were presented at ESICM Lives 2015 as an e-poster [42].

\section{Competing interests}

The authors declare that they have no competing interests. Medtronic Sentrino ${ }^{\circledR}$ provided two CGM systems for the duration of the study, but had no influence on study design and data analysis.

\section{Availability of data and materials}

Ethical restrictions prevent public sharing of data. Editors, reviewers and interested researchers should contact the corresponding author or dairesearchdata@charite.de to request data access.

\section{Consent for publication}

Informed consent included consent for publication.

\section{Ethics approval and consent to participate}

The local ethics committee, Charité-Universitätsmedizin Berlin Ethikkommission, approved the study (Charité-EA2/095/14). Informed consent was given by the patient or legal proxy.

\section{Funding}

This investigation was a preliminary study within the European Critical Care Research Network (ECCRN) Grant for Practice Improvement in ICU Nutrition 2013 given to TW. SWC received financial support from Deutsche Forschungsgemeinschaft (DFG) KFO 192 TP3. Medtronic provided two CGM monitor systems for the duration of the study, but had no role in study design, data collection and analysis, decision to publish, or preparation of the manuscript.

Received: 24 February 2016 Accepted: 28 June 2016

Published online: 21 July 2016

\section{References}

1. McCowen KC, Malhotra A, Bistrian BR. Stress-induced hyperglycemia. Crit Care Clin. 2001;17:107-24

2. Preiser J-C, Ichai C, Orban J-C, Groeneveld ABJ. Metabolic response to the stress of critical illness. Br J Anaesth. 2014;113:945-54.

3. Weber-Carstens S, Schneider J, Wollersheim T, Assmann A, Bierbrauer J, Marg A, et al. Critical illness myopathy and GLUT4: significance of insulin and muscle contraction. Am J Respir Crit Care Med. 2013;187:387-96

4. Krinsley JS. Association between hyperglycemia and increased hospital mortality in a heterogeneous population of critically ill patients. Mayo Clin Proc. 2003;78:1471-8

5. Krinsley JS. Glycemic variability: a strong independent predictor of mortality in critically ill patients*. Crit Care Med. 2008;36:3008-13.

6. NICE-SUGAR Study Investigators, Finfer S, Liu B, Chittock DR, Norton R, Myburgh JA, et al. Hypoglycemia and risk of death in critically ill patients. N Engl J Med. 2012;367:1108-18.

7. van den Berghe G, Wouters P, Weekers F, Verwaest C, Bruyninckx F, Schetz $M$, et al. Intensive insulin therapy in critically ill patients. N Engl J Med. 2001;345:1359-67.

8. Van den Berghe G, Wilmer A, Hermans G, Meersseman W, Wouters PJ, Milants I, et al. Intensive insulin therapy in the medical ICU. N Engl J Med. 2006;354:449-61.

9. NICE-SUGAR Study Investigators, Finfer S, Chittock DR, Su SY-S, Blair D, Foster $\mathrm{D}$, et al. Intensive versus conventional glucose control in critically ill patients. N Engl J Med. 2009;360:1283-97.

10. Marik PE. Toward understanding tight glycemic control in the ICU: a systematic review and metaanalysis. Chest J. 2010;137:544.
11. Wernerman J, Desaive T, Finfer S, Foubert L, Furnary A, Holzinger U, et al. Continuous glucose control in the ICU: report of a 2013 round table meeting. Crit Care. 2014;18:226

12. Aragon D. Evaluation of nursing work effort and perceptions about blood glucose testing in tight glycemic control. Am J Crit Care. 2006;15:370-7.

13. Boom DT, Sechterberger MK, Rijkenberg S, Kreder S, Bosman RJ, Wester JP, et al. Insulin treatment guided by subcutaneous continuous glucose monitoring compared to frequent point-of-care measurement in critically ill patients: a randomized controlled trial. Crit Care. 2014 [cited 2015 Sep 12];18. http://www.ncbi.n/m.nih.gov/pmc/articles/PMC4161875/.

14. Holzinger U, Warszawska J, Kitzberger R, Wewalka M, Miehsler W, Herkner $\mathrm{H}$, et al. Real-time continuous glucose monitoring in critically ill patients. Diabetes Care. 2010;33:467-72.

15. Brunner R, Kitzberger R, Miehsler W, Herkner H, Madl C, Holzinger U. Accuracy and reliability of a subcutaneous continuous glucose-monitoring system in critically ill patients*. Crit Care Med. 2011;39:659-64.

16. Sechterberger MK, van der Voort PHJ, Strasma PJ, DeVries JH. Accuracy of intra-arterial and subcutaneous continuous glucose monitoring in postoperative cardiac surgery patients in the ICU. J Diabetes Sci Technol. 2015;9:663-7.

17. van Hooijdonk RT, Leopold JH, Winters T, Binnekade JM, Juffermans NP, Horn J, et al. Point accuracy and reliability of an interstitial continuous glucose-monitoring device in critically ill patients: a prospective study. Crit Care. 2015 [cited 2015 Sep 12];19. http://www.ncbi.nlm.nih.gov/pmc/ articles/PMC4382831/.

18. Kosiborod M, Gottlieb RK, Sekella JA, Peterman D, Grodzinsky A, Kennedy $P$, et al. Performance of the medtronic sentrino continuous glucose management (CGM) system in the cardiac intensive care unit. BMJ Open Diabetes Res Care. 2014 [cited 2015 Sep 12];2. http://www.ncbi.nlm.nih. gov/pmc/articles/PMC4212554/.

19. Punke MA, Decker C, Wodack K, Reuter DA, Kluge S. Continuous glucose monitoring on the ICU using a subcutaneous sensor. Med Klin Intensivmed Notfallmedizin. 2015;110:360-3.

20. Finfer S, Wernerman J, Preiser J-C, Cass T, Desaive T, Hovorka R, et al. Clinical review: consensus recommendations on measurement of blood glucose and reporting glycemic control in critically ill adults. Crit Care. 2013:17:229.

21. Hill NR, Oliver NS, Choudhary P, Levy JC, Hindmarsh P, Matthews DR. Normal reference range for mean tissue glucose and glycemic variability derived from continuous glucose monitoring for subjects without diabetes in different ethnic groups. Diabetes Technol Ther. 2011;13:921-8

22. Bland JM, Altman DG. Comparing methods of measurement: why plotting difference against standard method is misleading. Lancet. 1995;346:1085-7.

23. Clarke WL, Cox D, Gonder-Frederick LA, Carter W, PohI SL. Evaluating clinical accuracy of systems for self-monitoring of blood glucose. Diabetes Care. 1987;10:622-8.

24. Klonoff DC, Lias C, Vigersky R, Clarke W, Parkes JL, Sacks DB, et al. The surveillance error grid. J Diabetes Sci Technol. 2014;8:658-72.

25. Schierenbeck F, Franco-Cereceda A, Liska J. Evaluation of a continuous blood glucose monitoring system using central venous microdialysis. J Diabetes Sci Technol. 2012;6:1365-71.

26. van Hooijdonk RT, Winters T, Fischer JC, van Dongen-Lases EC, Krinsley JS, Preiser J-C, et al. Accuracy and limitations of continuous glucose monitoring using spectroscopy in critically ill patients. Ann Intensive Care. 2014; $4: 8$.

27. Crane BC, Barwell NP, Gopal P, Gopichand M, Higgs T, James TD, et al. The development of a continuous intravascular glucose monitoring sensor. J Diabetes Sci Technol. 2015;9:751-61.

28. Macken L, Flower OJ, Bird S, Hammond N, Yarad E, Bass F, et al. Continuous intra-arterial blood glucose monitoring using quenched fluorescence sensing in intensive care patients after cardiac surgery: phase II of a product development study. Crit Care Resusc J Australas Acad Crit Care Med. 2015;17:190-6.

29. Basu A, Dube S, Slama M, Errazuriz I, Amezcua JC, Kudva YC, et al. Time lag of glucose from intravascular to interstitial compartment in humans. Diabetes. 2013;62:4083-7.

30. Scuffi C, Lucarelli F, Valgimigli F. Minimizing the impact of time lag variability on accuracy evaluation of continuous glucose monitoring systems. J Diabetes Sci Technol. 2012;6:1383-91. 
31. Rebrin K, Sheppard NF, Steil GM. Use of subcutaneous interstitial fluid glucose to estimate blood glucose: revisiting delay and sensor offset. J Diabetes Sci Technol. 2010;4:1087-98.

32. Boyne MS, Silver DM, Kaplan J, Saudek CD. Timing of changes in interstitial and venous blood glucose measured with a continuous subcutaneous glucose sensor. Diabetes. 2003;52:2790-4.

33. Cengiz E, Tamborlane WV. A tale of two compartments: interstitial versus blood glucose monitoring. Diabetes Technol Ther. 2009;11:S-11-6.

34. Lorencio C, Leal Y, Bonet A, Bondia J, Palerm CC, Tache A, et al. Real-time continuous glucose monitoring in an intensive care unit: better accuracy in patients with septic shock. Diabetes Technol Ther. 2012;14:568-75.

35. Holzinger U, Warszawska J, Kitzberger R, Herkner H, Metnitz PGH, Madl C. Impact of shock requiring norepinephrine on the accuracy and reliability of subcutaneous continuous glucose monitoring. Intensive Care Med. 2009;35:1383-9.

36. Siegelaar SE, Barwari T, Hermanides J, van der Voort PHJ, Hoekstra JBL, DeVries JH. Microcirculation and its relation to continuous subcutaneous glucose sensor accuracy in cardiac surgery patients in the intensive care unit. J Thorac Cardiovasc Surg. 2013;146:1283-9.
37. Igarashi M, Yamatani K, Fukase N, Daimon M, Ohnuma H, Takahashi H, et al. Sepsis inhibits insulin-stimulated glucose transport in isolated rat adipocytes. Diabetes Res Clin Pract. 1992;15:213-8.

38. Jansson PA, Fowelin J, Smith U, Lonnroth P. Characterization by microdialysis of intracellular glucose level in subcutaneous tissue in humans. Am J Physiol Endocrinol Metab. 1988;255:E218-20.

39. Rebrin K, Steil GM. Can interstitial glucose assessment replace blood glucose measurements? Diabetes Technol Ther. 2000;2:461-72.

40. Brunner R, Adelsmayr G, Herkner H, Madl C, Holzinger U. Glycemic variability and glucose complexity in critically ill patients: a retrospective analysis of continuous glucose monitoring data. Crit Care. 2012;16:R175.

41. Martin Bland J, Altman D. Statistical methods for assessing agreement between two methods of clinical measurement. Lancet. 1986;327:307-10

42. Engelhardt L, Wollersheim T, Pachulla J, Mörgeli R, Balzer F, Mai K, WeberCarstens S. Accuracy of a subcutaneous continuous glucose management system in critically ill patients. Intensive Care Med Exp. 2015;3(Suppl 1):A291.

\section{Submit your manuscript to a SpringerOpen ${ }^{\circ}$ journal and benefit from:}

- Convenient online submission

Rigorous peer review

- Immediate publication on acceptance

- Open access: articles freely available online

- High visibility within the field

- Retaining the copyright to your article 